\title{
Cell entry of one-dimensional nanomaterials occurs by tip recognition and rotation
}

\author{
Xinghua Shi ${ }^{1 \dagger}$, Annette von dem Bussche ${ }^{2}$, Robert H. Hurt ${ }^{1,3}$, Agnes B. Kane ${ }^{2,3 \star}$ and Huajian Gao ${ }^{1,3 \star}$
}

\begin{abstract}
Materials with high aspect ratio, such as carbon nanotubes and asbestos fibres, have been shown to cause length-dependent toxicity in certain cells because these long materials prevent complete ingestion and this frustrates the cell ${ }^{1-3}$. Biophysical models have been proposed to explain how spheres and elliptical nanostructures enter cells ${ }^{4-8}$, but one-dimensional nanomaterials have not been examined. Here, we show experimentally and theoretically that cylindrical one-dimensional nanomaterials such as carbon nanotubes enter cells through the tip first. For nanotubes with end caps or carbon shells at their tips, uptake involves tip recognition through receptor binding, rotation that is driven by asymmetric elastic strain at the tube-bilayer interface, and near-vertical entry. The precise angle of entry is governed by the relative timescales for tube rotation and receptor diffusion. Nanotubes without caps or shells on their tips show a different mode of membrane interaction, posing an interesting question as to whether modifying the tips of tubes may help avoid frustrated uptake by cells.
\end{abstract}

Understanding the way cells interact with one-dimensional nanomaterials is important for creating safer biomedical diagnostics and therapies ${ }^{9,10}$, and for regulating occupational and environmental exposure ${ }^{1,3,11}$. One-dimensional structures represent an important class of nanomaterials, whose cellular interactions are particularly complex and have been associated with the induction of inflammation, fibrosis and malignant mesothelioma ${ }^{1-3,11,12}$. Incomplete or frustrated phagocytosis of long, rigid biopersistent asbestos fibres or carbon nanotubes is hypothesized to lead to prolonged generation of reactive oxygen species, leading to the release of inflammatory mediators, cell death ${ }^{11,13}$ and to DNA and chromosomal damage in target cells of the lung and pleura ${ }^{12,14}$. DNA and chromosomal damage induced by asbestos fibres or carbon nanotubes may contribute to the development of lung cancer and malignant mesothelioma following inhalation ${ }^{11,12}$.

Fibre-cell interaction begins with membrane contact, adhesion and uptake. Biophysical models have been developed for the endocytosis or phagocytosis ${ }^{4-8,15}$ of nanoparticles, including models for the treatment of elliptical particles ${ }^{5,15}$. Here we focus on one-dimension nanomaterials, that is, those with a very high aspect ratio, focusing on the important case of cylindrical symmetry, which includes carbon nanotubes, asbestos fibres and gold nanowires. In previous studies ${ }^{3}$, and in our preliminary studies using in situ spinning disk confocal microscopy with dual fluorescence and polarized light imaging, multi-walled carbon nanotubes (MWCNT) were observed to enter cells tip first, but the mechanism was unclear, because optical microscopy offers insufficient resolution to reveal nanostructural features at the tube-membrane interface. Here we use ex situ field-emission scanning electron microscopy (SEM) following fixation and contrast enhancement with osmium tetroxide to obtain nanoscale resolution. We focus on liver cells and mesothelial cells because they are important targets for carbon nanotube exposure following intravenous injection ${ }^{1,16,17}$, or inhalation and translocation to the mesothelial lining of the pleural space surrounding the lungs ${ }^{17}$.

Figure 1 shows electron micrographs of common morphologies in the near-membrane region following in vitro cell exposure to one-dimensional nanomaterials. Carbon nanotubes undergoing active entry are primarily found protruding at a high angle (Fig. 1a,b), consistent with our preliminary polarized light confocal images. Figure 1a shows high-magnification images of the nanotubes at the point of entry showing invagination of the plasma membrane and the absence of a protruding phagocytic cup, which are characteristic morphological feature of endocytosis. An endocytic entry mechanism is not surprising, as neither of these cell types is a professional phagocyte, and the MWCNT tips have diameters of $50-100 \mathrm{~nm}$, which is within the favourable size range for receptor-mediated endocytosis of particles ${ }^{4}$. To determine whether uptake of carbon nanotubes is mediated by energy-dependent endocytosis, murine liver cells were incubated at either $4{ }^{\circ} \mathrm{C}$ or $37^{\circ} \mathrm{C}$. Internalization of carbon nanotubes was significantly decreased at $4{ }^{\circ} \mathrm{C}$ (Fig. 1d). In the presence of metabolic inhibitors, uptake of carbon nanotubes was also significantly decreased (Fig. 1d) confirming that this uptake requires adenosine triphosphate (ATP) (Supplementary Figs 1, 2).

In addition to the structures in Fig. 1, we observe nanotubes lying flat on the membrane, but these show no visible signs of active uptake or membrane response (Supplementary Fig. 3a). Uncommon structures include nanotubes entering at low angle or lying flat with active membrane wrapping in the horizontal configuration (Supplementary Fig. 3b). Tip entry is observed to be the dominant uptake mode, not only for MWCNTs, but also for other one-dimensional materials tested here (Fig. 1c), amine-terminated gold nanowires and crocidolite asbestos fibres.

We became interested in the fundamental question of why tip entry is the preferred mode of cellular uptake. Based on previous modelling of endocytosis for spheres, we hypothesized that nanotube tips with closed, rounded caps can mimic particles and initiate endocytosis, and that elastic strain in the plasma membrane provides a driving force to rotate one-dimensional nanostructures from their initial angle of contact to high angles that minimize total membrane elastic energy. To test this hypothesis, we began coarse-grained molecular dynamics (CGMD) simulations of receptor-mediated endocytosis of capped MWCNTs entering through a dipalmitoylphosphatidylcholine (DPPC) model lipid bilayer (Fig. 2). The simulation includes a population of diffusible membrane-bound receptors. The specific receptors that may recognize high-aspect-ratio nanoparticles include Class A or Class B

\footnotetext{
'School of Engineering, Brown University, Providence, Rhode Island 02912, USA, ²Department of Pathology and Laboratory Medicine, Brown University, Providence, Rhode Island 02912, USA, ${ }^{3}$ Institute for Molecular and Nanoscale Innovation, Brown University, Providence, Rhode Island, 02912, USA; †Present address: State Key Laboratory of Nonlinear Mechanics, Institute of Mechanics, Chinese Academy of Sciences, Beijing 100190, China. ^e-mail: Huajian_Gao@brown.edu; Agnes_Kane@brown.edu
} 

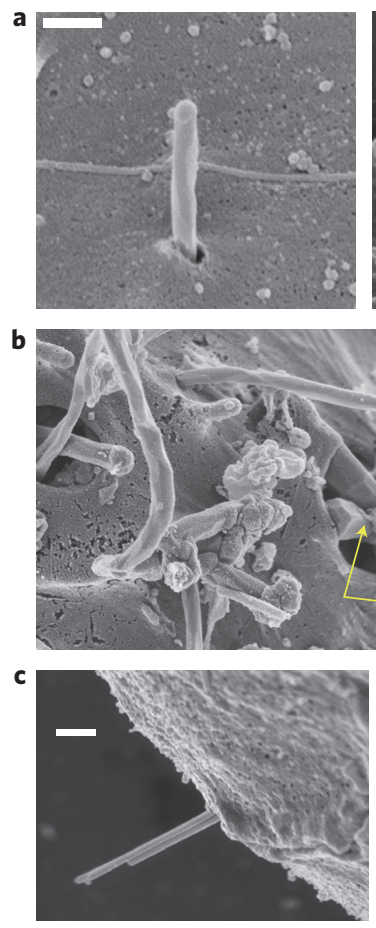
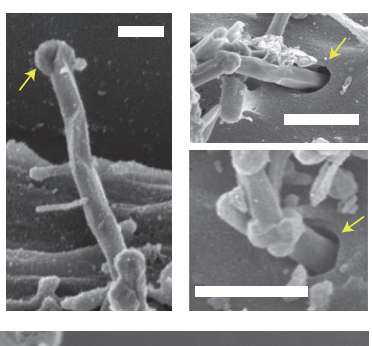
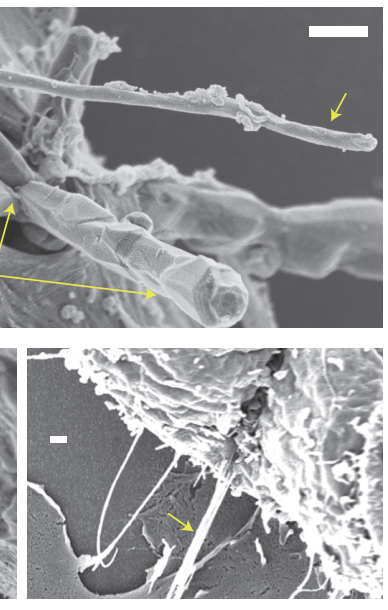

Figure 1 | Experimental evidence for energy-dependent tip-entry mode in the cellular interactions of one-dimensional nanomaterials. a-c, Field-emission SEM images following fixation and osmium tetroxide staining. Scale bars, $300 \mathrm{~nm}$. a, Entry of MWCNTs into murine liver cells. Left: MWCNT undergoing high-angle tip entry. Middle: arrow shows carbon shell that distinguishes nanotubes from surface microvilli. Right: arrows show membrane invaginations at the point of entry, characteristic of endocytosis. b, Isolated MWCNT (single arrow) and tube bundle (double arrow) entering human mesothelial cells at high angle. c, Gold nanowires (30 nm, left) and $500 \mathrm{~nm}$ crocidolite asbestos fibre (right) entering mesothelial cells through their tips. d, Reduced uptake at $4{ }^{\circ} \mathrm{C}$ (top) and in the presence of a metabolic inhibitor mixture (bottom) containing $\mathrm{NaF}_{\text {, }} \mathrm{NaN}_{3}$ and antimycin $\mathrm{A}$. Error bars show standard error; asterisks indicate that the temperature and inhibitor effects are statistically significant $(P<0.0005$ and 0.005 , respectively), confirming energy-dependent endocytosis.

scavenger receptors that are expressed primarily on macrophages and bind asbestos fibres ${ }^{18}$ as well as $\mathrm{MWCNTs}^{19}$. Other receptors expressed by epithelial cells that bind to MWCNTs include lectin receptors ${ }^{20}$, a variety of which are known to be expressed by liver cells $^{21,22}$. Asbestos fibres also bind vitronectin, a serum protein that binds to negatively charged surfaces and is recognized by integrin receptors on both mesothelial ${ }^{23}$ and lung epithelial cell ${ }^{24}$.

For the initial simulations, a capped MWCNT was initially positioned in close proximity above the surface of a bilayer with 16,326 lipids and receptors with dimensions of $100 \mathrm{~nm} \times 100 \mathrm{~nm}$. The initial angle between the axis of the MWCNT and the bilayer was $\theta_{0}=45^{\circ}$, and a range of receptor densities, $\phi$, were considered. As shown in Fig. 3a, when $\phi=0.25$, the receptors (green) diffuse along the bilayer and aggregate around the MWCNT due to binding affinity. As the receptors cluster and adhere to the MWCNT surface, the tube is pulled into the bilayer and wrapped. In this process the tube is observed to rotate to achieve an entry angle close to $90^{\circ}$. Rotation towards vertical alignment has also been reported by Yang and $\mathrm{Ma}^{7}$ in simulations of transmembrane diffusion of ellipsoidal nanoparticles. The present result shows similar behaviour for receptor-mediated entry of one-dimensional nanomaterials, and we will show shortly that the rotation is driven by membrane elastic energy minimization during wrapping.

Figure $3 \mathrm{~b}$ shows that at a higher receptor density of $\phi=0.33$, the MWCNT becomes fully wrapped before it reaches the $90^{\circ}$ entry angle. Generally, increasing the receptor density leads to increasing arrival flux of receptors at the tube surface (Supplementary Figs 4, 5 and discussion). In the extreme case of $\phi=1$, in which the adhesion loses specificity, the membrane on the right side of the nanotube adheres to the tube much faster than that on the left side (Fig. 3c), and the MWCNT adopts a very small entry angle. This can be understood from the fact that, for non-specific adhesion, the right-side membrane has the advantage of being initially closer to the tube surface and dominates the early-stage receptor binding before rotation can occur. These simulations reveal two competing kinetic processes: rotation of the tube toward a $90^{\circ}$ entry angle to relax elastic energy in the membrane,

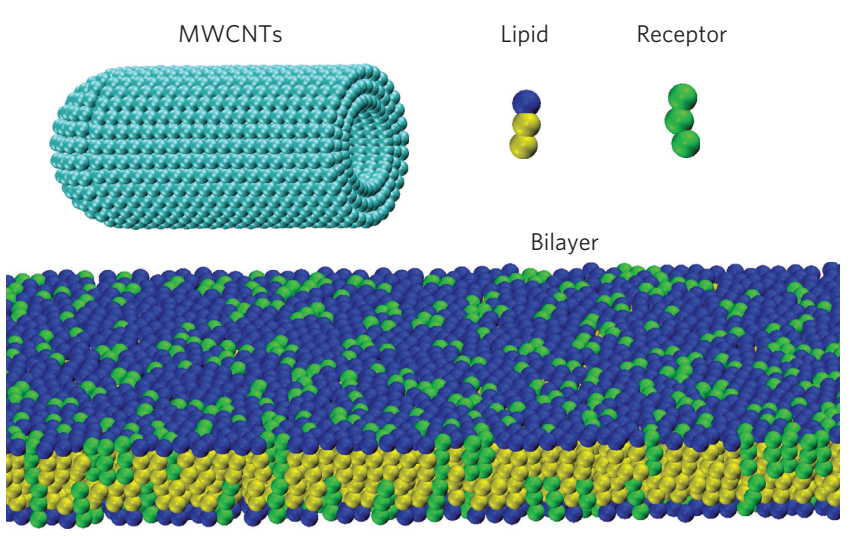

Figure 2 | Course-grained molecular dynamics simulation model. Models of DPPC lipid and receptor molecules formed by one hydrophilic head-bead and two hydrophobic tail-beads, and a capped MWCNT with diameter $d=20 \mathrm{~nm}$ and length $L=46 \mathrm{~nm}$ consisting of three concentric walls. A membrane bilayer consisting of lipid and receptor molecules spans the simulation box. 
a
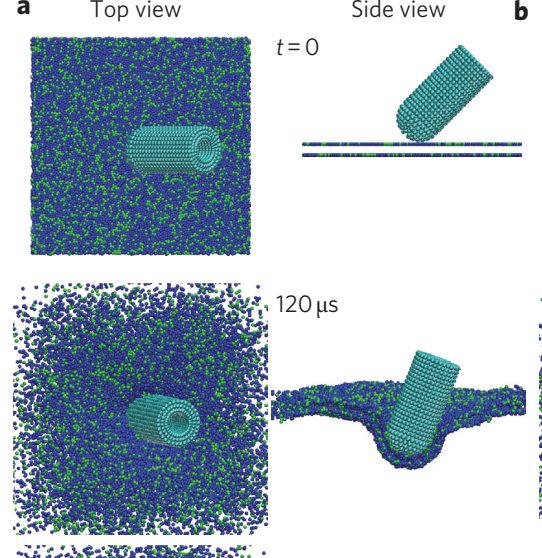

\section{b}
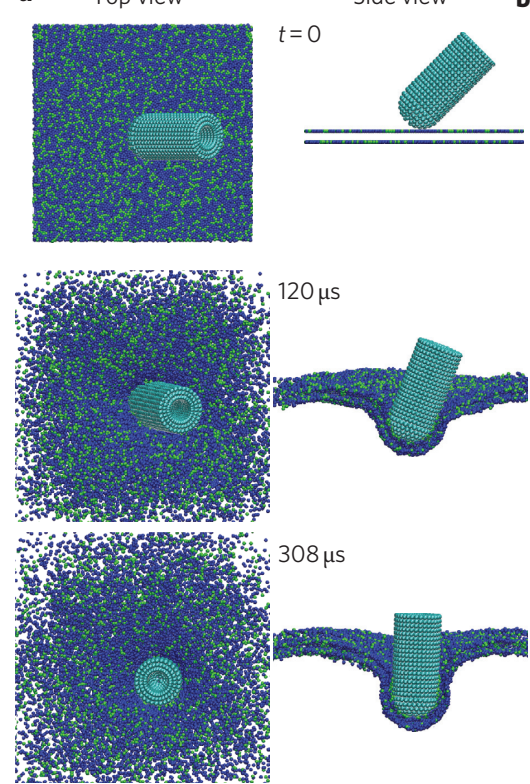
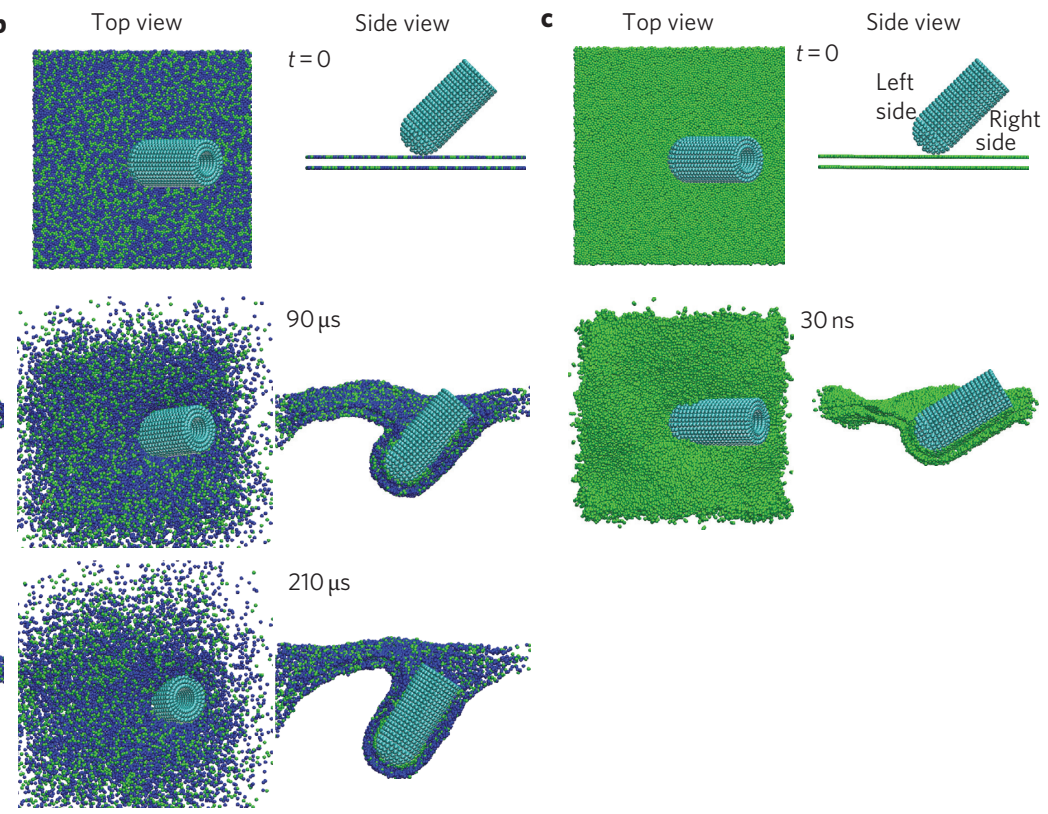

Figure 3 | Time sequence of CGMD simulation results showing a MWCNT penetrating the cell membrane at an initial entry angle of $\boldsymbol{\theta}_{0}=45^{\circ}$. a, At receptor (green) density $\phi=0.25$, the MWCNT rotates to $90^{\circ}$ before being fully wrapped. $\mathbf{b}$, At receptor density $\phi=0.33$, the tube is fully wrapped before reaching the $90^{\circ}$ entry angle. c, At receptor density $\phi=1$, the tube rotates towards a low entry angle.

and wrapping speeds on different sides of the tube governed by receptor diffusion. If the former prevails, as would be expected at relatively low receptor densities, the final entry angle will be close to $90^{\circ}$. Note that the extreme case of non-specific interactions shown in Fig. 3c is an interesting theoretical limit, which is not expected to be important for a real cellular system.

We repeated the simulations for different MWCNT diameters and lengths, receptor densities, receptor binding strengths and initial entry angles, $\theta=15^{\circ}$ or $85^{\circ}$, and the results show that the tube still adopts a $90^{\circ}$ entry pathway (Supplementary Figs 7-11). Further additional simulations show that the tip-entry mechanism is essentially unchanged if the hemispherical caps are replaced by enlarged shells typical of catalytically produced MWCNTs (as in Fig. 1a, centre), or if the nanotubes exist in suspension as small bundles (Supplementary Figs 6, 11). Interestingly, we observe that open-ended nanotubes do not undergo tip entry because they lack carbon atom sites for receptor binding on the cap in the early stages of wrapping (Supplementary Fig. 6c).

These CGMD simulations suggest that a biophysical mechanism drives the tip-entry pathway for cellular uptake of MWCNTs. The simulations further suggest that the steady-state entry angle is determined by the competition between speed of tip rotation and that of receptor binding to side walls during uptake. As a result of the wellknown limitations of molecular dynamics simulations, the wrapping timescale in a real cellular system cannot be reached (on the scale of minutes), even though, in the present CGMD simulations, the wrapping speed has already been accelerated by choosing relatively high receptor densities as well as smaller model receptors with faster diffusivity (see Supplementary Information). In addition, the viscosity of the cell cytosol, which could be orders of magnitude larger than that of the extracellular matrix ${ }^{25}$ and can greatly influence the rotating speed of MWCNT, is not correctly captured by the CGMD model. In this sense, the timescales in our CGMD simulations can only be used in a qualitative sense to gain insights into the tip-entry mechanism.

It would be useful to have a simple analytical model to complement the simulations and reveal fundamental scaling laws. Here, we develop a simplified model that is amenable to analytical solutions, following previous studies on receptor-mediated endocytosis ${ }^{4}$. It has been shown ${ }^{4}$ that the time for receptor-mediated wrapping of a CNT can be expressed as $t_{\mathrm{w}}=L_{\mathrm{i}} d / 4 \alpha^{2} D$, where $D$ is the diffusivity of receptors in the membrane, $d$ is the tube diameter, $L_{\mathrm{i}}$ is the length of the tube that has been wrapped into the membrane, and $\alpha$ is a 'speed factor' (see Methods). Taking typical parameter values as $D \approx 1 \times 10^{3}-1 \times 10^{4} \mathrm{~nm}^{2} \mathrm{~s}^{-1}, e_{\mathrm{RL}}=15, B=$ $20, \xi_{\mathrm{L}}=5,000 \mu \mathrm{m}^{-2}, \tilde{\xi}=0.1, d=20 \mathrm{~nm}$ (ref. 4), the predicted wrapping time is shown in Fig. $4 \mathrm{a}$ as a function of the engulfed length of the MWCNT. The results show that $20 \mathrm{~s}$ will be needed to take in a 30- $\mu \mathrm{m}$-long nanotube for $D=1 \times 10^{4} \mathrm{~nm}^{2} \mathrm{~s}^{-1}$ (Fig. 4a, blue line) and $200 \mathrm{~s}$ for $D=1 \times 10^{3} \mathrm{~nm}^{2} \mathrm{~s}^{-1}$ (Fig. 4a, red line).

The tip rotation of a MWCNT during uptake is attributed to a torque $T$ exerted by the curved bilayer on a partially wrapped tube and resisted by viscous drag on the rotating MWCNT in the extracellular and intracellular spaces (see Methods for derivation). Figure $4 \mathrm{~b}$ shows the calculated dynamics of MWCNT rotation during uptake at different values of initial entry angle $\theta_{0}$. It is seen that the MWCNT adjusts to a steady-state entry angle within a few seconds. The decreasing slope of the evolution profile indicates that the speed of tip rotation decreases as the tube is wrapped into the cell. For low initial entry angles (for example, $\theta_{0}=15^{\circ}$ ), the slope of tip-angle evolution is larger than that at high entry angles (for example, $\theta_{0}=75^{\circ}$ ), indicating that the rotational speed is entry angle dependent, which is consistent with our CGMD simulations. At very small initial entry angles, such as $\theta_{0}=1^{\circ}$, the MWCNT exhibits a strong tendency to rotate to a large entry angle. Compared to the characteristic timescale for full uptake ( 20-200 s), the timescale for tip rotation (4s) is one or two orders of magnitude smaller. Because the tip rotation is much faster than overall wrapping, tip entry is a favourable pathway for cellular uptake of capped MWCNTs.

The above simulation results and analysis support our experimental observations of tip entry, but the analysis has been limited to finite positive initial angles of attack. In the extreme case of $0^{\circ}$ approach, in which case the entire sidewall of a MWCNT comes into contact with the membrane at once, the 

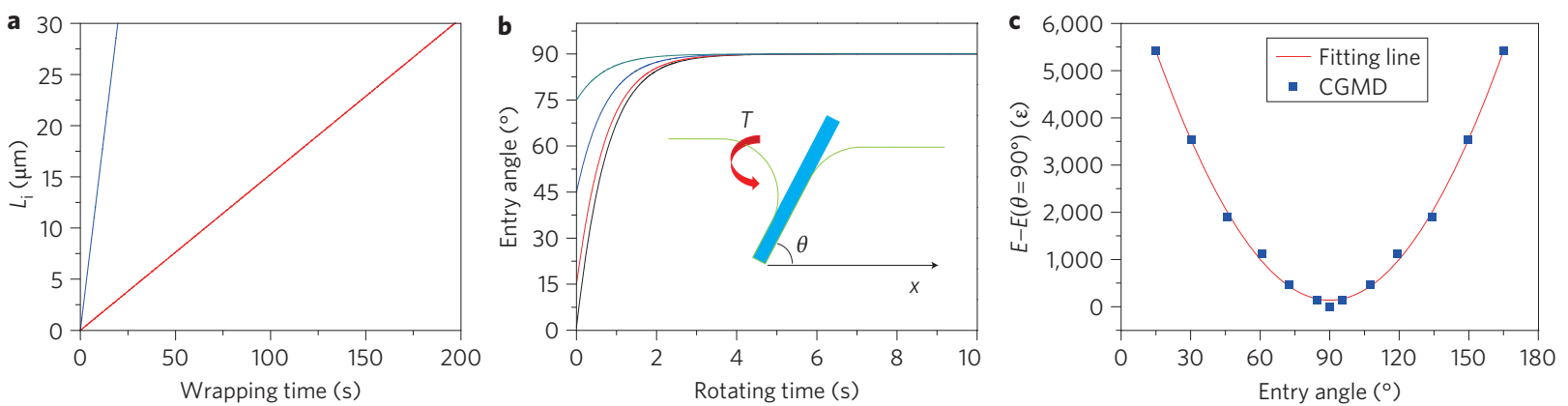

Figure 4 | Analytical model of a MWCNT entering cell. $\mathbf{a}, \mathbf{b}$, Timescales associated with wrapping and tip rotation: entry time as a function of the engulfed length (a) under selected parameter values $e_{\mathrm{RL}}=15, B=20, \xi \mathrm{L}=5,000 \mu \mathrm{m}^{-2}, \tilde{\xi}=0.1, d=20 \mathrm{~nm}, D=1 \times 10^{3}$ (red line) or $1 \times 10^{4} \mathrm{~nm}^{2} \mathrm{~s}^{-1}$ (blue line); evolution of the entry angle of the MWCNT (b) at different initial entry angles $\theta_{0}=1^{\circ}, 15^{\circ}, 45^{\circ}$ and $75^{\circ}$. The other parameters are $d=20 \mathrm{~nm}, L=L_{\mathrm{i}}+L_{\mathrm{e}}=$ $30 \mu \mathrm{m}, \eta_{\mathrm{e}}=1 \times 10^{-3} \mathrm{~Pa} \mathrm{~s}, \eta_{\mathrm{i}}=10 \mathrm{~Pa}$ s. Inset to $\mathbf{b}$ : schematic of an MWCNT entering a cell at angle $\theta$. The curved bilayer exerts torque Tonto the MWCNT. c, Total elastic energy as a function of the entry angle of the MWCNT relative to its reference value at the entry angle of $90^{\circ}$. The solid red line is a fit from CGMD simulation results (blue squares).

nanotubes seem to attach to the membrane without visible signs of active uptake, as shown in Supplementary Fig. 3a. We propose that this phenomenon is due to receptor depletion caused by the large contact area. Following previous studies on receptor-mediated endocytosis $^{4}$, for a membrane patch $20 \mu \mathrm{m}$ in length, the maximum diameter of MWCNTs that can be fully wrapped is $\sim 64 \mathrm{~nm}$, when the ratio between receptor density and binding sites on MWCNTs is taken to be $\tilde{\xi}=0.01$. Therefore, the receptors can be easily depleted by a $0^{\circ}$ wrapping due to the large contact area. The receptor depletion would become even more pronounced in the presence of bundles or ensembles of MWCNTs in horizontal contact with cell membrane patches (Supplementary Fig. 3a).

We note that the tip-entry mode provides a natural physicomechanical explanation for incomplete endocytosis or frustrated phagocytosis, which plays an important role in human health risk. Why do cells initiate uptake of one-dimensional structures that are in fact too long for complete internalization? In our model, receptor clustering at the rounded tube caps initiates uptake of what appears to be a particle, followed by a relatively rapid strain-induced transition to vertical or near-vertical fibre alignment. This vertical geometry provides no opportunity for the cell membrane to sense or anticipate the ultimate length of the fibrous target material. If the target fibre is too long for successful internalization and subsequent packaging in endosomes, then the initial wrapping and intake will lead inevitably to incomplete endocytosis.

A mechanistic link between incomplete uptake of high-aspectratio nanoparticles and length-dependent toxicity has been proposed by Hamilton et al. ${ }^{26}$ In macrophages exposed to $\mathrm{TiO}_{2}$ nanorods, incomplete uptake of long nanorods $(>15 \mu \mathrm{m})$ resulted in a failure to sequester nanorods within lysosomes. Subsequently, release of cathepsin B-a lysosomal protease-activates the NALP3 inflammasome and production of proinflammatory mediators. Disruption of the normal process of endosomal-lysosomal fusion has also been shown to lead to cell death by apoptosis following activation of caspase-1 by cathepsin B in macrophages following exposure to asbestos fibres ${ }^{27}$. Incomplete endocytosis of high-aspect-ratio nanoparticles by liver cells can similarly lead to failure of sequestration into lysosomes, with subsequent physical interference with cytoskeletal-mediated processes, including protein and lipid secretion and biliary transport.

Finally, our modelling work suggests that open-tipped tubes exhibit a very different mode of membrane interaction. Intelligent tip modification may provide new approaches in the future to engineer one-dimensional nanomaterials for cell delivery, or to avoid frustrated uptake and achieve material safety by design.

\section{Methods}

Materials and dispersion method. The experimental portion of this study focuses on the interaction of three high-aspect-ratio nanomaterials with two immortalized, non-tumorigenic cell lines (mouse AML12 liver cells and human LP9 mesothelial cells). MWCNTs (MER Corporation) were synthesized by chemical vapour deposition (CVD), and had a typical length of $13 \mu \mathrm{m}$, diameter of $85 \mathrm{~nm}$, total surface area of $20 \mathrm{~m}^{2} \mathrm{~g}^{-1}$ determined by molecular nitrogen vapour adsorption and analysis by the Brunauer-Emmett-Teller (BET) theory and contained $0.2 \mathrm{wt} \% \mathrm{Fe}$ catalyst residue. They were used as-received without covalent surface modification, and exhibited zeta potentials of -20 to $-30 \mathrm{mV}$ in cell culture medium. Crocidolite asbestos fibres were purchased from Duke Scientific and ranged from 0.1 to $35.1 \mu \mathrm{m}$ in length and 30-3,000 $\mathrm{nm}$ in diameter, with a BET surface area of $9 \mathrm{~m}^{2} \mathrm{~g}^{-1}$. The amine-terminated gold nanowires were purchased from Nanoparz and were $\sim 6 \mu \mathrm{m}$ in length and $30 \mathrm{~nm}$ in diameter. The test materials were dispersed in $1 \%$ fetal calf serum (FCS) containing Dulbecco's modified Eagle medium: nutrient mixture F-12 (DMEM/F12) at a concentration of $1,000 \mu \mathrm{g} \mathrm{ml}^{-1}$. Following sonication $(100 \mathrm{~W})$ in a Branson 2510 sonicating bath for $20 \mathrm{~min}$, the stock was diluted to a final concentration of $500 \mu \mathrm{g} \mathrm{ml}^{-1}$ and sonicated for an additional $20 \mathrm{~min}$.

Cell studies. Mouse AML12 liver cells (ATTC number, CRL-225) were cultured in DMEM/F12 medium containing 10\% FCS, $0.005 \mathrm{mg} \mathrm{ml}^{-1}$ insulin, $0.005 \mathrm{mg} \mathrm{ml}^{-1}$ transferrin, $5 \mathrm{ng} \mathrm{ml}^{-1}$ selenium, and $40 \mathrm{ng} \mathrm{ml}^{-1}$ dexamethasone in cell culture flasks at $37^{\circ} \mathrm{C}$. After removing the cell culture medium, liver cells were briefly rinsed with $0.25 \%(\mathrm{w} / \mathrm{v})$ Trypsin-0.53 mM ethylenediaminetetraacetic acid (EDTA) solution and incubated for $5 \mathrm{~min}$ in Trypsin- $0.53 \mathrm{mM}$ EDTA solution at $37^{\circ} \mathrm{C}$. Complete growth medium was added, and cells were plated onto glass cover slips in six-well plates (diameter/well, $35 \mathrm{~mm}$ ). The cells were allowed to attach overnight at $37^{\circ} \mathrm{C}$ and exposed with $20 \mu \mathrm{g} \mathrm{ml}^{-1}$ test material for an additional $24 \mathrm{~h}$.

The LP9 mesothelialline is an hTERT-immortalized normal human peritoneal cell line (obtained from J. Rheinwald at the Brigham and Women's Hospital Cell Culture Core in Boston, MA). Human mesothelial cells were cultured in a mixture of M199 and MCDB105 medium containing 15\% FCS, $0.4 \mu \mathrm{g} \mathrm{ml}^{-1}$ hydrocortisone and $10 \mathrm{ng} \mathrm{ml}^{-1}$ epidermal growth factor (EGF) in cell culture flasks at $37^{\circ} \mathrm{C}$. After removing the cell culture medium, the cells were briefly rinsed with trypsin $0.05 \%$ containing EDTA in Hanks' balanced salt solution, and incubated for $1 \mathrm{~min}$ in $0.05 \%$ trypsin at $37^{\circ} \mathrm{C}$. Complete growth medium was added, and cells were plated onto cover glass slips in six-well plates (diameter/well, $35 \mathrm{~mm}$ ). The cells were allowed to attach overnight at $37^{\circ} \mathrm{C}$ and were exposed to $20 \mu \mathrm{g} \mathrm{ml}^{-1}(20 \mathrm{ppm})$ test material suspension for an additional $24 \mathrm{~h}$.

CNT-exposed cells were fixed in Karnovsky's fixative (5\% glutaraldehyde, $4 \%$ formaldehyde in $0.1 \mathrm{M}$ sodium cacodylate buffer, $\mathrm{pH}$ 7.4: Electron Microscopy Sciences, \# 11650) for 2 days at $4{ }^{\circ} \mathrm{C}$, then rinsed three times with $0.1 \mathrm{M}$ sodium cacodylate buffer. The fixed cells were post-fixed in $2 \%$ aqueous osmium tetroxide (Electron Microscopy Sciences, \#RT 19152) for $30 \mathrm{~min}$ followed by dehydration in $25 \%, 50 \%, 70 \%, 2 \times 95 \%, 3 \times 100 \%$ ethanol. After critical point drying according to the manufacturer's directions (Ladd Research), the cover slips were sputter-coated with gold and viewed by field-emission SEM (LEO 1530-VP).

To test for energy-dependent endocytosis ${ }^{28}$, murine liver cells were seeded and allowed to attach to 16 -well glass slides (Lab Tek) overnight. Cells were placed at $4{ }^{\circ} \mathrm{C}$ for $30 \mathrm{~min}$ and exposed to $80 \mathrm{ppm}$ carbon nanotubes for $2 \mathrm{~h}$. The control group was exposed to $80 \mathrm{ppm}$ carbon nanotubes for $2 \mathrm{~h}$ at $37^{\circ} \mathrm{C}$. A further test for the endocytosis mechanism involved metabolic inhibition experiments. Here, cells were pre-incubated in cell culture medium containing $0.02 \%$ sodium azide, $1.875 \mathrm{mM}$ sodium fluoride and $0.25 \mu \mathrm{g} \mathrm{ml}^{-1}$ antimycin A for $30 \mathrm{~min}$, followed by exposure to $80 \mathrm{ppm}$ carbon nanotubes in cell culture medium containing the metabolic 
inhibitors. The cells were washed and stained with anti-tubulin antibody and 4',6diamidino-2-phenylindole (DAPI). Images were visualized using an Olympus confocal microscope (IX81 motorized inverted research microscope) with polarized light filters to image uptake. Quantitation of carbon nanotube uptake was performed using MetaMorph Microscopy Automation \& Image Analysis Software 6.3. Cells exposed to metabolic inhibitors for $2.5 \mathrm{~h}$ were assessed for ATP content using a luminescence-based ATP assay (Invitrogen). Cell viability in both experiments was confirmed using Pico Green (Invitrogen) to quantitate DNA content.

Coarse-grained molecular simulations. CGMD simulations based on the simulation code ESPRESSO ${ }^{29}$ were performed to investigate the tip entry of capped MWCNTs into a cell membrane consisting of DPPC lipid molecules. Each DPPC lipid molecule was modelled as three connected beads with one hydrophilic headbead and two hydrophobic tail-beads (Fig. 2). To construct the bilayer with an appropriate thickness, the bead diameter $\sigma$ was set to $1 \mathrm{~nm}$. As shown in Fig. 2, the coarse-grained model of capped MWCNTs is constructed by three SWCNTs with all the nearest and second-nearest beads connected by harmonic springs with spring constant $k=400 \varepsilon$. The nearest-neighbour distance of the beads on the outermost surface of CNTs was $2 \sigma$. Detailed interactions among the beads are provided in the Supplemental Information. The CGMD simulations were performed under constant temperature $\left(k_{\mathrm{B}} T=1.1 \varepsilon\right)$ and pressure with a dissipative particle dynamics (DPD) thermostat. A damping parameter $\Gamma=1.0 \varepsilon \tau / \sigma^{2}$ and a cut-off distance $d_{\text {cut }}=3.0 \sigma$ were used in the thermostat. A barostat with damping parameter $\Gamma_{\mathrm{A}}=0.0001 \varepsilon \tau / \sigma^{4}$, piston mass $M=0.0005 \varepsilon \tau^{2} / \sigma^{4}$ and zero external pressure was used to control the lateral tension of the bilayer by altering the dimensions of the simulation box size along the bilayer. A similar procedure has been previously used to investigate the aggregation and vesiculation of membrane proteins ${ }^{29}$.

Determination of speed factor $\boldsymbol{\alpha}$. The speed factor $\alpha$ was determined from the governing equation

$$
e_{\mathrm{RL}}-\frac{1}{2} B \frac{1}{(d / 2)^{2} \xi_{L}}+\ln f(\alpha)-f(\alpha)+1=0
$$

which originates from the balance of energies during uptake ${ }^{4}$. Here, $e_{\mathrm{RL}} k_{\mathrm{B}} T$ is the binding energy of a single receptor-ligand bond, $B k_{\mathrm{B}} T$ is the bending modulus of the membrane

$$
f(\alpha)=\tilde{\xi}+\frac{\alpha^{2}(1-\tilde{\xi}) E_{1}\left(\alpha^{2}\right)}{\alpha^{2} E_{1}\left(\alpha^{2}\right)-\mathrm{e}^{-\alpha^{2}}}
$$

$\tilde{\xi}=\xi_{0} / \xi_{\mathrm{L}}, \xi_{0}$ and $\xi_{\mathrm{L}}$ are the densities of receptor and ligand, and

$$
E_{1}(z)=\int_{z}^{\infty} \mathrm{e}^{-u} / u \mathrm{~d} u
$$

In the present model, we found that the curvature of the membrane and the radius of MWCNTs have only a minor influence on the speed factor $\alpha$. In the analysis, $\alpha$ is assumed to be identical on both sides of the MWCNT.

\section{Development of dynamic rotation model. In a model of membrane-CNT} interaction, an energetic torque can be calculated from the variation of elastic energy as $T=-\mathrm{d} E / \mathrm{d} \theta$, where $E$ is the elastic energy at a fixed tube-membrane contact area. A fully three-dimensional theoretical expression of the total elastic energy is beyond the scope of the present work. We extracted the elastic energy directly from the CGMD simulations using the empirical fitting function (see Fig. 4c)

$$
E=E\left(\theta=90^{\circ}\right)+28,407 \varepsilon\left[1+\sin \left(\frac{9}{19} \theta+\frac{24}{19} \pi\right)\right]
$$

The torque depends on the entry angle $\theta$. The smaller the entry angle, the larger the torque. At the nanoscale, the intra/extracellular environment is considered a lowReynolds-number medium where the inertia force can be ignored, so the applied force would be balanced by a drag force. The rotating angular velocity is then expressed as ${ }^{30}$

$$
\frac{\mathrm{d} \theta}{\mathrm{d} t}=\frac{3 T}{4 \pi\left[\frac{\eta_{\mathrm{i}} L_{\mathrm{i}}^{3}}{C_{\mathrm{i}}}+\frac{\eta_{\mathrm{e}} L_{\mathrm{e}}^{3}}{C_{\mathrm{e}}}\right]}
$$

where $\eta_{\mathrm{i}}$ and $\eta_{\mathrm{e}}$ are the viscosities of the intra- and extracellular environments, respectively, $L_{\mathrm{i}}=4 \alpha^{2} D t / d$ and $L_{\mathrm{e}}$ are the lengths of the nanotube inside and outside the membrane, $C_{\mathrm{i}}=\ln p_{\mathrm{i}}-0.662+0.917 / p_{\mathrm{i}}-0.05 / p_{\mathrm{i}}^{2}$ with $p_{\mathrm{i}}=L_{\mathrm{i}} / d$ where $d$ is the tube diameter, and $C_{\mathrm{e}}=\ln p_{\mathrm{e}}-0.662+0.917 / p_{\mathrm{e}}-0.05 / p_{\mathrm{e}}^{2}$ with $p_{\mathrm{e}}=L_{\mathrm{e}} / d$. The differential equation is then solved with $d=20 \mathrm{~nm}, L=L_{\mathrm{i}}+L_{\mathrm{e}}=30 \mu \mathrm{m}$, $\eta_{\mathrm{e}}=1 \times 10^{-3} \mathrm{~Pa} \mathrm{~s}, \eta_{\mathrm{i}}=10 \mathrm{~Pa} \mathrm{~s}$.
Received 4 April 2011; accepted 15 August 2011;

published online 18 September 2011

\section{References}

1. Poland, C. A. et al. Carbon nanotubes introduced into the abdominal cavity of mice show asbestos-like pathogenicity in a pilot study. Nature Nanotech. $\mathbf{3}$, 423-428 (2008).

2. Donaldson, K., Murphy, F. A., Duffin, R. \& Poland, C. A. Asbestos, carbon nanotubes and the pleural mesothelium: a review of the hypothesis regarding the role of long fibre retention in the parietal pleura, inflammation and mesothelioma. Part. Fibre Toxicol. 7, 5 (2010).

3. Brown, D. M. et al. An in vitro study of the potential of carbon nanotubes and nanofibres to induce inflammatory mediators and frustrated phagocytosis. Carbon 45, 1743-1756 (2007).

4. Gao, H. J., Shi, W. D. \& Freund, L. B. Mechanics of receptor-mediated endocytosis. Proc. Natl Acad. Sci. USA 102, 9469-9474 (2005).

5. Decuzzi, P. \& Ferrari, M. The receptor-mediated endocytosis of nonspherical particles. Biophys. J. 94, 3790-3797 (2008).

6. Chen, H., Langer, R. \& Edwards, D. A. A film tension theory of phagocytosis. J. Colloid Interface Sci. 190, 118-133 (1997).

7. Yang, K. \& Ma Y. Q. Computer simulation of the translocation of nanoparticles with different shapes across a lipid bilayer. Nature Nanotech. 5, 579-583 (2010).

8. Zhang, S., Li, J., Lykotrafitis, G., Bao, G. \& Suresh, S. Size-dependent endocytosis of nanoparticles. Adv. Mater. 21, 419-424 (2009).

9. Kostarelos, K., Bianco, A. \& Prato, M. Promises, facts and challenges for carbon nanotubes in imaging and therapeutics. Nature Nanotech. 4, 627-633 (2009).

10. Cheung, W., Pontoriero, F., Taratula, O., Chen, A. M. \& He, H. DNA and carbon nanotubes as medicine. Adv. Drug. Deliv. Rev. 62, 633-649 (2010).

11. Sanchez, V. C., Pietruska, J. R., Miselis, N. R., Hurt, R. H. \& Kane, A. B. Biopersistence and potential adverse health impacts of fibrous nanomaterials: what have we learned from asbestos? Wiley Interdiscip. Rev. Nanomed. Nanobiotechnol. 5, 511-529 (2009).

12. Nagai, H. \& Toyokuni, S. Biopersistent fiber-induced inflammation and carcinogenesis: lessons learned from asbestos toward safety of fibrous nanomaterials. Arch. Biochem. Biophys. 502, 1-7 (2010).

13. Unfried, K. et al. Cellular responses to nanoparticles: target structures and mechanisms. Nanotoxicology 1, 52-71 (2007).

14. MacCorkle, R. A., Sluttery, S. D., Nash, D. R. \& Brinkley, B. R. Intracellular protein binding to asbestos induces aneuploidy in human lung fibroblasts. Cell Motil. Cytoskel. 63, 646-657 (2006).

15. Champion, J. A. \& Mitragotri, S. Role of target geometry in phagocytosis. Proc. Natl Acad. Sci. USA 103, 4930-4934 (2006).

16. Yang, S. et al. Long-term accumulation and low toxicity of single-walled carbon nanotubes in intravenously exposed mice. Toxicol. Lett. 181, 182-189 (2008).

17. Porter, D. W. et al. Mouse pulmonary dose and time course responses induced by exposure to multi-walled carbon nanotubes. Toxicology 269, 136-147 (2010).

18. Resnick, D., Freedman, N. J., Xu, S. \& Krieger, M. Secreted extracellular domains of macrophage scavenger receptors form elongated trimers, which specifically bind crocidolite asbestos. J. Biol. Chem. 5, 3538-3545 (1993).

19. Hirano, S., Kanno, S. \& Furuyama, A. Multi-walled carbon nanotubes injure the plasma membrane of macrophages. Toxicol. Appl. Pharmacol. 232, 244-251 (2008).

20. Zhang, L. W. \& Monteiro-Riviere, N. A. Lectins modulate multi-walled carbon nanotubes cellular uptake in human epidermal keratinocytes. Toxicol. Vitro 24, 546-551 (2010)

21. Wu, J., Nantz, M. H. \& Zern, M. A. Targeting hepatocytes for drug and gene delivery: emerging novel approaches and applications. Front. Biosci. 7, 717-725 (2002).

22. Moghimi, S. M. \& Hunter, A. C. Recognition by macrophages and liver cells of opsonized phospholipid vesicles and phospholipid headgroups. Pharmacol. Res. 18, 1-8 (2001).

23. Wu, J., Liu, W., Koenig, K., Idell, S. \& Broaddus, V. C. Vitronectin adsorption to chrysotile asbestos increases fiber phagocytosis and toxicity for mesothelial cells. Am. J. Physiol. Lung Cell Mol. Physiol. 279, L916-L923 (2000).

24. Pande, P., Mosleh, T. A. \& Aust, A. E. Role of alpha v beta 5 integrin receptor in endocytosis of crocidolite and its effect on intracellular glutathione levels in human lung epithelial (A 549) cells. Toxicol. Appl. Pharmacol. 210, 70-77 (2006).

25. Nelson, P. Biological Physics: Energy, Information, Life (Freeman, 2003).

26. Hamilton, R. F. Jr et al. Particle length-dependent titanium dioxide nanomaterials toxicity and bioactivity. Part. Fibre Toxicol. 6, 35 (2009).

27. Dostert, C. et al. Innate immune activation through Nalp3 inflammasome sensing of asbestos and silica. Science 320, 674-677 (2008).

28. $\mathrm{Xu}$, Y. et al. Nona-Arginine facilitates delivery of quantum dots into cells via multiple pathways. J. Biomed. Biotechnol. 2010, 948543 (2010).

29. Reynwar, B. J. et al. Aggregation and vesiculation of membrane proteins by curvature-mediated interactions. Nature 447, 461-464 (2007).

30. Tirado, M. M., Martinez, C. L. \& de la Torre, J. G. Comparison of theories for the translational and rotational diffusion coefficients of rod-like macromolecules. Application to short DNA fragments. J. Chem. Phys. 81, 2047-2052 (1984). 


\section{Acknowledgements}

This work was supported by the National Science Foundation (NSF; grant CMMI1028530), the US Department of Commerce, National Institute of Standards and Technology as part of the Rhode Island Consortium for Nanoscience and Nanotechnology, the National Institute of Environmental Health Sciences (NIEHS) Superfund Research Program P42 ES013660, and an R01 grant (ES016178) from the NIEHS. The simulations reported were performed on NSF TeraGrid resources provided by National Institute for Computational Sciences (NICS; under MCB090194) and resources from the Supercomputing Center of Chinese Academy of Sciences (SCCAS) and Shanghai Supercomputer Center (SSC). The authors thank P. Weston in the Molecular Pathology Core at Brown University for her assistance with electron microscopic sample preparation and imaging and F. Guo for measurement of MWCNT zeta potentials.

\section{Author contributions}

X.H.S., A.v.d.B., R.H.H., A.B.K. and H.J.G. conceived and designed the experiments and simulations. X.H.S. performed the simulations. A.v.d.B. performed the experiments. X.H.S., A.v.d.B., R.H.H., A.B.K. and H.J.G. analysed the data. X.H.S., A.v.d.B., R.H.H., A.B.K. and H.J.G. co-wrote the paper. All authors discussed the results and commented on the manuscript.

\section{Additional information}

The authors declare no competing financial interests. Supplementary information accompanies this paper at www.nature.com/naturenanotechnology. Reprints and permission information is available online at http://www.nature.com/reprints. Correspondence and requests for materials should be addressed to A.B.K. and H.G. 